\title{
Taxocenose de serpentes no Planalto Médio do Rio Grande do Sul, Brasil
}

\author{
Noeli Zanella ${ }^{1} \&$ Sonia Z. Cechin ${ }^{2}$
}

\begin{abstract}
${ }^{1}$ Instituto de Ciências Biológicas, Universidade de Passo Fundo. Caixa Postal 611/631, 99001-970, Passo Fundo, Rio Grande do Sul, Brasil. E-mail: zanella@upf.br
\end{abstract}

2 Departamento de Biologia, Centro de Ciências Naturais e Exatas, Universidade Federal de Santa Maria. Faixa de Camobi, Km 9, Camobi, 97105-900. Santa Maria, Rio Grande do Sul, Brasil. E-mail: cechinsz@ccne.ufsm.br

\begin{abstract}
Taxocenosis of snakes in the middle plateau region of Rio Grande do Sul, Brazil. The present study was conducted on the Brigada Farm (28 $14^{\prime} 39^{\prime \prime}$, 52 $\left.2^{\circ} 4^{\prime} 42^{\prime \prime} \mathrm{W}\right)$, Passo Fundo, middle plateau region of Rio Grande do Sul. The objective of the study was to provide data regarding the richness and abundance of snakes and to compare sampling methods. The study was carried out in two areas, i.e., forest and field, between January 2001 and March 2003. Data were collected using the following methods: time-constrained search (TCS), occasional encounters by local collectors (OEC), occasional encounters by the team (OET), and by pitfall traps (PT). Using the four methods, 19 snake species belonging to three families (Colubridae, Elapidae and Viperidae) were recorded, and 284 individuals were collected, including 128 by OEC, 36 by TCS, 112 by PT, and 8 by OET. Taking the results of all four methods into account, the three most abundant species were Thamnodynastes strigatus (Günther, 1858) (16.9\%), Echinanthera cyanopleura (Cope, 1885) (15.2\%) and Bothrops alternatus Duméril, Bibron 8 Duméril, 1854 (13.4\%). The study of this taxocenosis reveals important characteristics of the ecology of snakes and may contribute to future conservation studies in this area.
\end{abstract}

KEY WORDS. Community; diversity; methods.

RESUMO. Este trabalho foi desenvolvido na Fazenda da Brigada (28¹'39"S, 52 $14^{\prime} 42^{\prime \prime}$ W), Passo Fundo, Planalto Médio do Rio Grande do Sul, e teve como objetivo contribuir com informações sobre riqueza, abundância de serpentes e comparação entre os métodos de amostragem. O estudo foi desenvolvido em duas áreas, floresta e campo, no período de janeiro de 2001 a março de 2003. Para a coleta de dados foram utilizados os seguintes métodos: procura limitada por tempo (PLT), encontros ocasionais por terceiros (EOT), encontros ocasionais pela equipe (EOE) e armadilhas de interceptação e queda (AQ). Com a utilização dos quatro métodos foi possível registrar 19 espécies de serpentes, distribuídas em três famílias (Colubridae, Elapidae e Viperidae) e coletar 284 indivíduos, sendo 128 por EOT, 36 por PLT, 112 por AQ e 8 por EOE. Utilizando todos os métodos, as três espécies mais abundantes foram: Thamnodynastes strigatus (Günther, 1858) $(16,9 \%)$, Echinanthera cyanopleura $($ Cope, 1885) $(15,2 \%)$ e Bothrops alternatus Duméril, Bibron \& Duméril, $1854(13,4 \%)$. O estudo dessa taxocenose revela importantes características da ecologia de serpentes e poderá subsidiar futuros estudos de conservação para a área.

PALAVRAS CHAVE. Comunidades; diversidade; métodos.

Nos últimos anos esforços têm sido concentrados na tentativa de entender as interações intra e interespecíficas em determinadas comunidades, porém ainda há muito a compreender. O que se percebe é que o estudo de taxocenoses de serpentes está muito aquém de outros vertebrados, como peixes, lagartos, aves e mamíferos (Turner 1977, VitT 1987). A ecologia de taxocenoses de serpentes permanece como um dos temas mais difíceis e pouco estudados (Price \& Lapointe 1990), e, conseqüentemente, mal compreendidos.

A maior parte dos estudos de história natural sobre serpentes foi desenvolvida em zona temperada, na América do Norte, e em área tropical, na América Central e Austrália (VITT 1987,
Seigel et al. 1987, Seigel \& Collins 1993, Price \& Lapointe 1990). A região neotropical apresenta alta biodiversidade de répteis Squamata, inclusive de serpentes. Estudos de história natural sobre serpentes contribuem de forma expressiva para aumentar os conhecimentos da biologia dos diferentes táxons e suas inter-relações dentro de comunidades. Ainda há poucos estudos sobre taxocenoses de serpentes, o que dificulta a compreensão dos fatores que atuam na sua estruturação e no estabelecimento de padrões dessas comunidades.

Os estudos sobre comunidades de serpentes no Brasil são recentes (VITT \& VANGILDER 1983, SAZIMA \& HADDAD 1992, STRÜsSMANN \& Sazima 1993, Sazima \& Manzani 1995, Martins \& Oliveira 1998, 
Strüssmann 2000, Marques \& Sazima 2004) e no sul do Brasil não existem trabalhos publicados sobre este assunto.

Dessa forma, este estudo pretende contribuir para ampliar, na região neotropical, o conhecimento sobre taxocenoses de serpentes. São fornecidas informações sobre a estrutura de uma taxocenose de serpentes no Planalto Médio do Rio Grande do Sul, principalmente composição, riqueza e abundância relativa das espécies, com comparações entre os métodos de amostragem, contribuindo com informações que são relevantes para subsidiar estratégias de conservação e manejo.

\section{MATERIAL E MÉTODOS}

\section{Área de estudo}

A área selecionada para o presente trabalho pertence à Fazenda da Brigada Militar $\left(28^{\circ} 14^{\prime} 39^{\prime \prime}\right.$, $\left.52^{\circ} 19^{\prime} 42^{\prime \prime} \mathrm{W}\right)$ e situase no município de Passo Fundo, Rio Grande do Sul (Fig. 1). A área é formada por floresta secundária alterada, com 112 ha e campo alterado, que compreende aproximadamente 100 ha, com presença de gado. Os dois ambientes apresentam altitudes variando entre 630 e $740 \mathrm{~m}$.

Dentre a variação de vegetação que ocorre no estado do Rio Grande do Sul, as florestas do município de Passo Fundo estão em uma zona de transição entre os pinhais dos Campos de Cima da Serra e as florestas da bacia do Uruguai, apresentando vegetação mista denominada floresta ombrófila mista (Quadros \& Pillar 1990), contendo em seus estratos inferiores elementos vegetais característicos do Planalto e da bacia do Alto Uruguai. Os campos dessa região caracterizam-se pela presença de gramíneas e leguminosas escassas (BARRETO \& BOLDRINI 1990).

Pela classificação de KöPpen (1948), o clima local é descrito como subtropical úmido, com chuvas bem distribuídas durante o ano e temperatura do mês mais quente superior a $22^{\circ} \mathrm{C}$ e a do mês mais frio oscilando entre -3 e $10^{\circ} \mathrm{C}$ (média anual de 16,9 a $18,4^{\circ} \mathrm{C}$ ). O regime pluviométrico oscila entre 1575 e 1986 $\mathrm{mm}$ anuais, com a umidade relativa variando de 73 a $79 \%$ (EMBRAPA 1994).

\section{Métodos de amostragem}

Os métodos de amostragem utilizados para a localização e captura das serpentes foram:

Procura limitada por tempo (PLT). Este método consistiu em caminhadas ao longo e nas imediações das áreas definidas para procura de serpentes (floresta e campo), procurando abranger o maior número possível de micro-habitats. As áreas foram exploradas visualmente, com inspeção de tocas, serapilheira, troncos caídos, vegetação aquática e galhos de árvores, até aproximadamente três metros de altura. O período de amostragem foi de janeiro de 2001 a fevereiro de 2003. A equipe (1-5 pessoas) e o número de horas de procura foram variáveis (uma a cinco horas cada turno), com um mínimo de 50 horas/observador de procura por mês, totalizando 26 meses e 1.128 horas/observador. O esforço de procura durante o dia foi de 1.016 e 112 horas du-

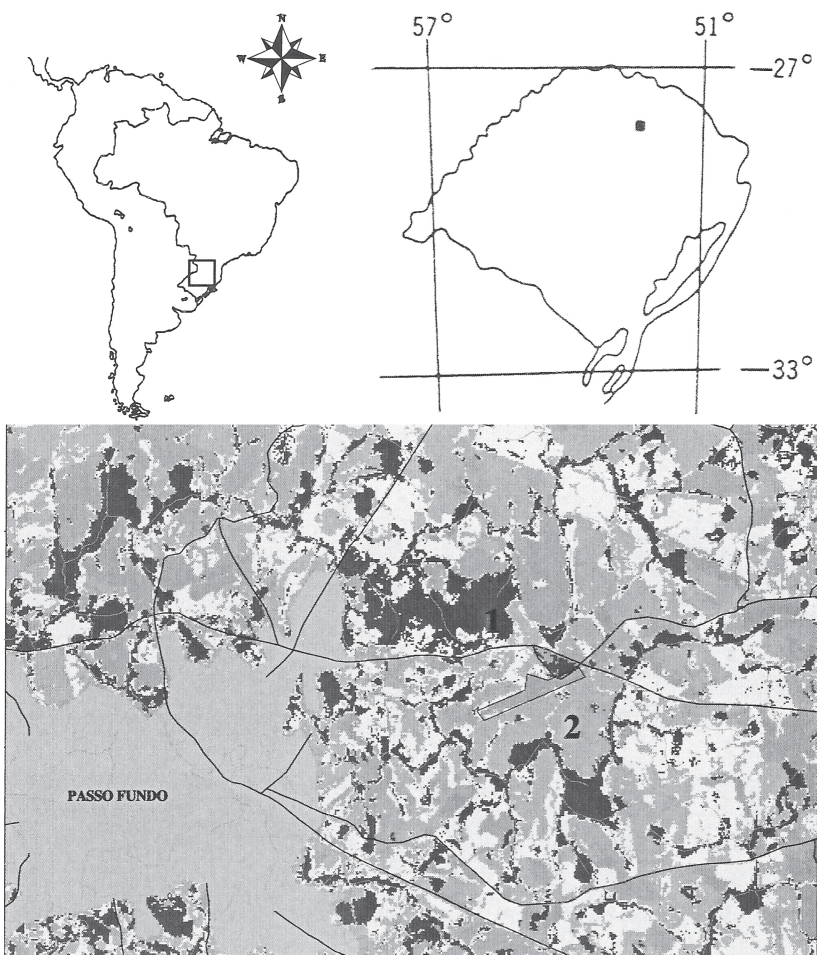

Figura 1. Área de estudo, Passo Fundo, Rio Grande do Sul: (1) floresta, (2) campo.

rante a noite. O esforço de procura foi considerado como o número de horas de atividade de procura de cada coletor. A taxa de encontro foi calculada da seguinte forma: o número de serpentes encontradas por hora-observador.

Encontros ocasionais (EO). Foram os encontros de animais antes ou depois da procura limitada por tempo durante o deslocamento, denominados encontros ocasionais da equipe (EOE), além de animais encontrados por terceiros na área de estudo (EOT). Moradores próximos à área receberam um balde contendo formol 10\% para acondicionar o material coletado. O período de amostragem utilizando este método foi de 24 meses.

Armadilhas de queda (AQ). As armadilhas de interceptação e queda com cerca-guia foram instaladas nas duas áreas de amostragem selecionadas. Foram instaladas duas séries de armadilhas em forma de " $\mathrm{T}$ ", compostas de 14 recipientes de plástico de 150 litros, sete em cada linha, instalados com um intervalo de $20 \mathrm{~m}$ cada um. A tela utilizada foi de aproximadamente $1 \mathrm{~m}$ de altura com $140 \mathrm{~m}$ em cada série, totalizando 560 m e 28 recipientes. As duas séries de armadilhas em cada área distaram cerca de $1000 \mathrm{~m}$ uma da outra. O período de amostragem foi de março de 2001 a março de 2003, totalizando 25 meses. Um total de 734 dias das armadilhas abertas correspondeu a 17.616 horas de amostragem.

Os indivíduos coletados foram incorporados à coleção 
de répteis da Universidade de Passo Fundo (CRUPF) (Anexo I). Para a identificação das serpentes foram utilizadas principalmente as referências de Peters \& Orejas-Miranda (1970), Dixon (1989), CEI (1993) e LEMA (1994).

\section{Análise de dados}

Foram utilizados os seguintes estimadores não paramétricos de riqueza de espécies: Jacknife de primeira ordem (Colwell \& Coddington 1994). A unidade amostral para as análises equivaleu a um mês para todos os métodos utilizados. Para a confecção de curvas de rarefação de espécies (sensu GotelLi \& Colwell 2001) e dos estimadores de riqueza, foi utilizado o programa Estimates 5.0 (Colwell 1997).

Para analisar a estrutura da taxocenose das duas áreas de estudo foram feitos gráficos de abundância relativa de espécies. Estes se baseiam na ordenação do logn da abundância das espécies em relação ao número das espécies (MagurRAN 1988).

\section{RESULTADOS E DISCUSSÃO}

\section{Riqueza e abundância}

No período de janeiro de 2001 a março de 2003 foram capturados 284 exemplares e registradas 19 espécies de serpentes distribuídas em três famílias e 12 gêneros, que correspondem a $84,2 \%$ da família Colubridae, 5,3\% de Elapidae e 10,5\% de Viperidae. A riqueza de espécies é semelhante nos dois ambientes estudados (campo, $\mathrm{n}=16$ táxons e floresta, $\mathrm{n}=14$ táxons) e, em relação à composição da taxocenose, $21 \%$ das espécies são exclusivas da floresta e $21 \%$ exclusivas do campo. Neste estudo, o campo apresentou a maior riqueza e menor dominância em relação à floresta, evidenciando maior diversidade (Figs 2 e 3). Diversos são os fatores que podem estar relacionados à riqueza de uma taxocenose. Padrões de riqueza de espécies de serpentes são complexos e influenciados por recursos disponíveis, pelo grau de especialização das espécies e sobreposição dos nichos, pela latitude, altitude e clima (ScotT 1976, VITT 1987). Os habitats alterados podem estar influenciando a menor riqueza de espécies na floresta, pois esse fragmento está isolado e é menor, quando comparado ao fragmento de campo. Ambos fragmentos estão inseridos numa matriz com plantio de culturas anuais. Em comunidades alteradas por atividades antrópicas, freqüentemente observa-se diminuição no número de espécies e aumento de dominância de algumas espécies (AcCACio et al. 2003).

Incluindo todos os métodos de amostragem, as três espécies mais abundantes nas duas áreas foram: Thamnodynastes strigatus (Günther, 1858) (16,9\%), Echinanthera cyanopleura (Cope, 1885) $(15,2 \%)$ e Bothrops alternatus Duméril, Bibron \& Duméril, 1854 (13,4\%) (Fig. 4). As espécies mais abundantes na floresta foram E. cyanopleura (30,9\%), T. strigatus $(24,5 \%)$ e Tomodon dorsatus Duméril, Bibron \& Duméril, 1854 (11,5\%). No campo foram B. alternatus $(16,6 \%)$, Liophis poecilogyrus (Wied, 1824) (13,8\%) e T. strigatus $(9,6 \%)$.

Considerando o método de encontro por terceiros, as três
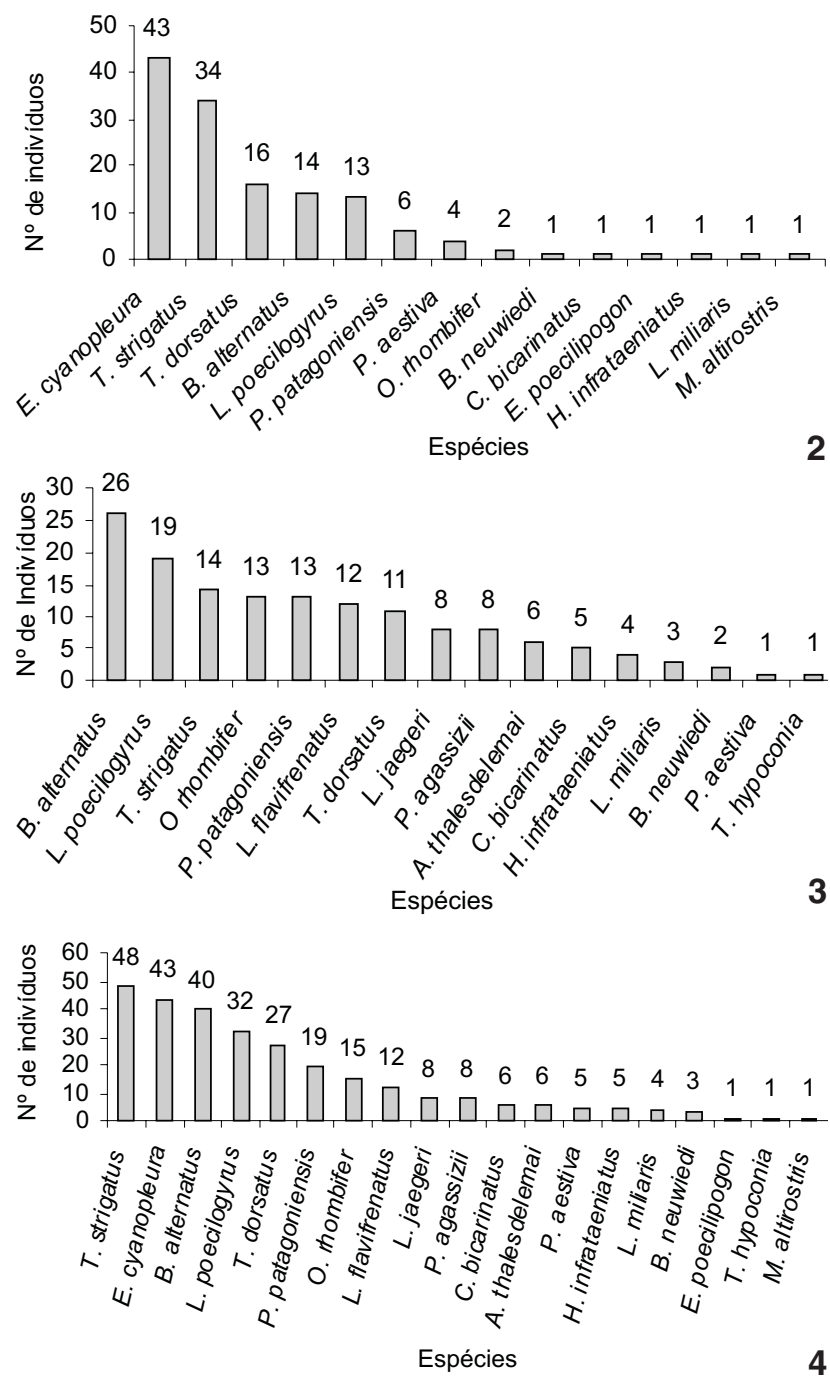

Figuras 2-4. Composição e abundância relativa das espécies de serpentes do Planalto Médio do Rio Grande do Sul, no período de janeiro de 2001 a março de 2003, considerando todos os métodos de amostragem: (2) na floresta, (3) na área de campo, (4) considerando floresta e campo. O número de indivíduos de cada espécie é indicado acima de cada barra.

espécies mais abundantes na floresta $(\mathrm{n}=54)$ foram Bothrops alternatus $(25,9 \%)$, Tomodon dorsatus $(20,4 \%)$ e Thamnodynastes strigatus $(16,7 \%)$. No campo $(\mathrm{n}=75)$ foram B. alternatus $(28 \%)$, Liophis poecilogyrus $(18,7 \%)$ e T. dorsatus (12\%). Bothrops alternatus foi a espécie mais abundante pelo método de encontro por terceiros nas duas áreas. Espécies de Bothrops Wagler, 1824 são as mais abundantes em vários biomas neotropicais, especialmente em área aberta (YANOSKY et al. 1996, SAZIMA \& HADDAD 1992). A plasticidade no uso do ambiente, a morfologia, o tamanho corporal e a dieta generalista podem ter facilitado o 
elevado sucesso da diversificação ecológica de Bothrops na América do Sul (MARTins et al. 2001). A maior abundância dessa espécie também deve estar associada ao método de encontros ocasionais por terceiros, pois a captura ocorre geralmente em ambientes alterados e está sujeita à tendenciosidade dos coletores residentes, que capturam em maior quantidade espécies conspícuas, menos propensas à fuga, com pelo menos parte de sua atividade durante o dia.

Considerando o método de armadilhas, na floresta $(\mathrm{n}=$ 61), as três espécies mais abundantes foram Echinanthera cyanopleura (55,7\%), Thamnodynastes strigatus $(22,9 \%)$ e Liophis poecilogyrus $(11,5 \%)$. No campo as mais abundantes $(\mathrm{n}=51)$ foram Oxyrhopus rhombifer Duméril, Bibron \& Duméril, 1854) (21,6\%), L. flavifrenatus (Cope, 1862) (21,6\%) e Pseudablabes agassizii (Jan, 1853) (17,6\%). Echinanthera cyanopleura é espécie exclusiva de ambiente florestado e mais abundante que as demais. Esse fato pode estar associado à dieta, já que o item preferencial da espécie, anfíbios, também é abundante na serapilheira da floresta, onde esta espécie forrageia (MARQUES \& SAZIMA 2004). No campo, uma das espécies mais abundantes, $L$. flavifrenatus é exclusiva de área aberta, evidenciando adaptação a este tipo de ambiente (GIRAUDo 2001).

\section{Comparação entre as áreas amostradas}

Com a utilização desses métodos, foi possível coletar 284 indivíduos, sendo 128 por EOT, 36 por PLT, 112 por AQ e 8 EOE (Tab. I).

Tabela I. Riqueza e abundância utilizando diferentes métodos na área de estudo, no período de janeiro de 2001 a março de 2003 (PLT) Procura limitada por tempo, (EOT) encontros ocasionais por terceiros, (EOE) encontros ocasionais pela equipe, (AQ) armadilhas de interceptação e queda, (Todos) todos os métodos.

\begin{tabular}{lcrcrc}
\hline & PLT & EOT & EOE & AQ & Todos \\
\hline Riqueza & 14 & 15 & 6 & 12 & 19 \\
Abundância & 36 & 128 & 8 & 112 & 284 \\
\hline
\end{tabular}

Foram utilizadas 1.128 horas/observador na procura visual (PLT), com uma taxa de encontro de serpentes de 0,031 por hora-observador de procura limitada por tempo, ou seja, uma serpente a cada 35 horas-observador. Na floresta foram amostradas 535 horas/observador de procura, com taxa de encontro de 0,031 por hora-observador, e no campo 592 horas/observador, com taxa de encontro de 0,025 serpentes por hora-observador. Com esse método foram capturadas 14 espécies (Echinanthera poecilopogon (Cope, 1860), e Thamnodynantes hypoconia (Cope, 1860), exclusivas por este método), seis na floresta $(42,8 \%)$ e 11 no campo $(78,6 \%)$. Este método foi responsável por amostrar $73,7 \%$ da riqueza total da área de estudo.

Com o uso de armadilhas de queda, a taxa média de captura foi de 0,15 serpentes/recipiente/mês, com um esforço total de 143 horas utilizadas na inspeção das armadilhas. A eficiência das armadilhas (AQ) na amostragem da abundância das serpentes foi semelhante na floresta $(0,08$ serpentes/recipientes/mês) e no campo (0,07 serpentes/recipientes/mês) (Tab. II). Um total de 734 dias das armadilhas abertas correspondeu a 17.616 horas e a captura de 12 espécies (uma exclusiva), sete na floresta $(58,3 \%)$ e oito no campo $(66,7 \%)$. Armadilhas de interceptação e queda são extremamente eficientes em amostragens de anfíbios e répteis (CECHIN \& MarTins 2000) e apresentaram desempenho satisfatório na amostragem de serpentes da área. Esse método foi responsável por $63,1 \%$ da riqueza observada de serpentes e possibilitou também amostragem uniforme, simultânea e constante entre as duas áreas, o que permite uma análise quantitativa satisfatória.

Tabela II. Número de indivíduos (N), esforço total de amostragem (horas-observador) e taxa de captura na área de campo e mata, utilizando o método de armadilhas de queda, no período de março de 2001 a março de 2003, no Planalto Médio, Rio Grande do Sul.

\begin{tabular}{lccc}
\hline Fisionomia & $\mathrm{N}$ & $\begin{array}{c}\text { Esforço de } \\
\text { amostragem }\end{array}$ & Serpentes/recipiente/mês \\
\hline Floresta & 14 & $535 \mathrm{~h} / \mathrm{o}$ & 0,08 \\
Campo & 16 & $593 \mathrm{~h} / \mathrm{o}$ & 0,07 \\
Mata + campo & 19 & $1.128 \mathrm{~h} / \mathrm{o}$ & 0,15 \\
\hline
\end{tabular}

Das 28 espécies de serpentes registradas para o Planalto Médio nos últimos dez anos (dados não publicados), 19 espécies foram encontradas na área de estudo, correspondendo a $68 \%$ do total. O método de PLT foi mais eficaz na área aberta, enquanto as armadilhas mostraram eficiência muito próxima, tanto no campo quanto na floresta. A dificuldade de encontro de serpentes em regiões florestais se deve à ocorrência de espécies inconspícuas ou com hábitos crípticos, vegetação densa e serapilheira no solo (DuELlman 1987). Espécies diurnas são mais propensas à fuga com a aproximação do coletor, e muitas espécies podem apresentar padrões de coloração crípticos, que dificultam sua detecção (MarTins \& Oliveira 1998).

Com o método de encontros ocasionais foram capturadas 15 espécies (Micrurus altirostris (Cope, 1860), exclusiva por este método), nove na floresta (56,3\%) e 13 no campo $(81,3 \%)$. Este método foi responsável por amostrar $84,2 \%$ da riqueza da área. Os encontros ocasionais da equipe resultaram em uma espécie na floresta e cinco no campo. Martins \& Oliveira (1998) atribuem grande importância aos encontros ocasionais, mas indicam a utilização da associação de outros métodos. De fato, como mostram os dados, há a necessidade do esforço conjunto de todos os métodos para amostrar a riqueza das serpentes do Planalto Médio.

\section{Estimativas de riqueza da comunidade}

A estimativa de riqueza pelo estimador Jacknife de primeira ordem, utilizando procura limitada por tempo (PLT) indica que ocorrem na área 20 espécies de serpentes. O mesmo 

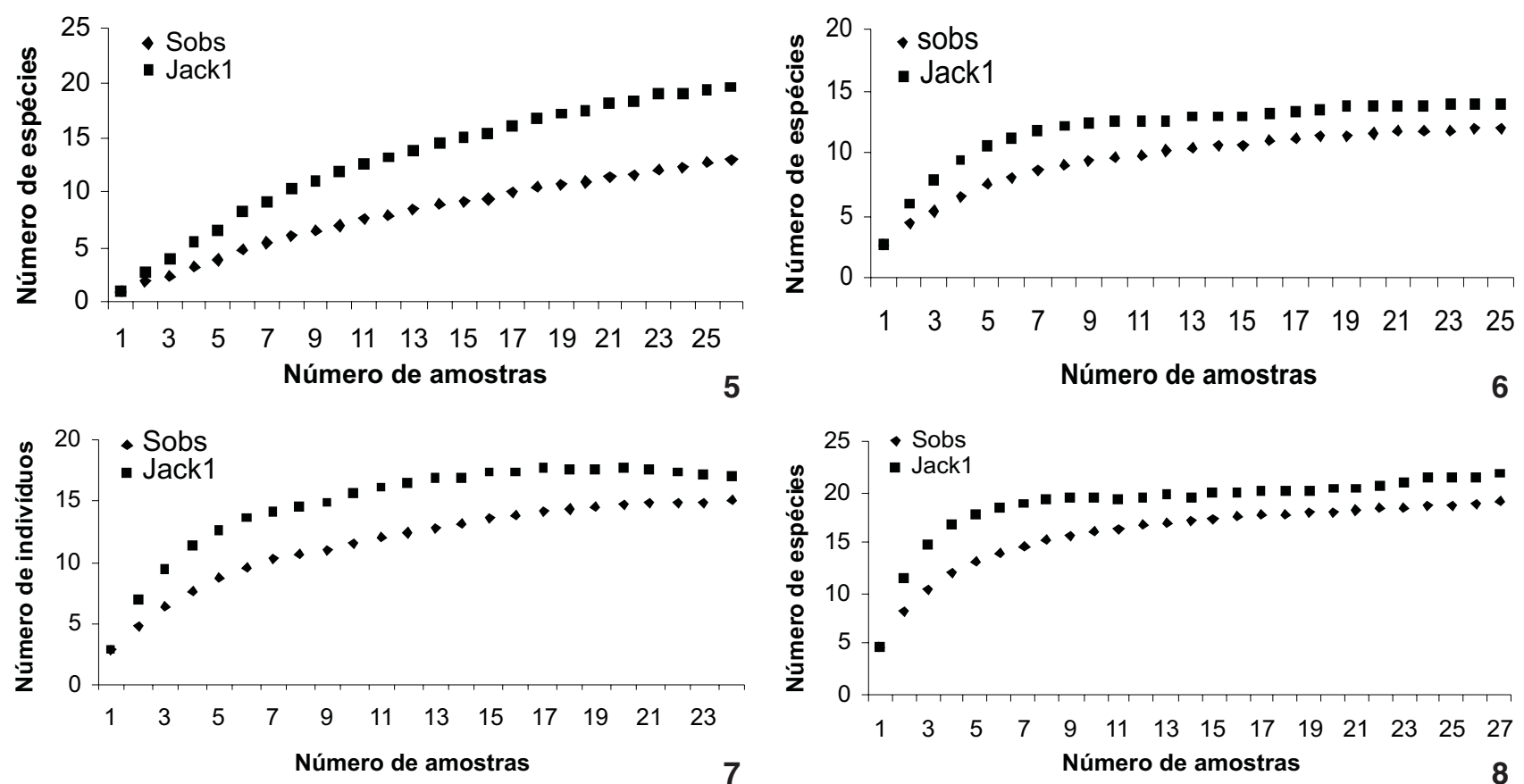

Figuras 5-8. Curvas de rarefação de espécies observadas (Sobs) e do estimador de riqueza Jacknife de primeira ordem (Jack1) utilizando o método: (5) procura limitada por tempo e 26 amostras; (6) armadilhas de queda e 25 amostras; (7) encontro por terceiros e 24 amostras; (8) todos os métodos e 27 amostras.

estimador de riqueza, para o método de armadilhas de queda (AQ) indica que ocorrem na área 14 espécies e para os encontros ocasionais de terceiros indica 20 espécies para a área. Considerado todos os métodos o mesmo estimador de riqueza indica que ocorrem na área 22 espécies para uma riqueza de 19 espécies observadas (Tab. III e Figs 5-8).

Tabela III. Sumário dos valores obtidos nas análises de riqueza de espécies, utilizando todos os métodos, armadilhas de queda (AQ), procura limitada por tempo (PLT) e encontros ocasionais por terceiros (EOT), para amostragem da taxocenose das serpentes, no Planalto Médio, Rio Grande do Sul.

\begin{tabular}{lcccc}
\hline \multicolumn{1}{c}{ Estimadores } & Todos & AQ & PLT & EOT \\
\hline Jacknife 1 & 22 & 14 & 20 & 20 \\
Riqueza observada & 19 & 12 & 13 & 16 \\
\hline
\end{tabular}

Todos os métodos apresentam limitações e o seu uso dificilmente permitiria capturar toda a riqueza da região. Curvas assintóticas assumem número finito de espécies capturáveis em uma determinada área, e quando esforço suficiente é aplicado, esse número de espécies poderia ser capturado (THOMPSON et al. 2003). As curvas sintóticas indicam que o número de indivíduos capturáveis poderia ser maior; entretanto, o aumento de esforço contribuiria muito lentamente com o aumento do número de espécies (THompson et al. 2003). A dificuldade de atingir a assíntota, no presente estudo, se deve ao tempo limitado de coleta e às limitações impostas pelos métodos utilizados, além do fato de serpentes apresentarem um número relativamente baixo de capturas. Desta forma, fica evidente a necessidade do aumento amostral para elevar a riqueza observada da área de estudo.

Xenodontíneos sul-americanos estão pobremente representados fora da América do Sul, com territórios muitos bem localizados, resultando em muitas espécies endêmicas (CADLE \& Greene 1993). A taxocenose do Planalto Médio é composta principalmente por xenodontíneos e mostra as tendências na distribuição geográfica da família Colubridae na região neotropical, com declínio de colubríneos e xenodontíneos centro-americanos à medida que analisamos a composição meridional (CADLE \& Greene 1993). De fato, a única linhagem que foi bem representada é a dos xenodontíneos da América do Sul, com 13 espécies (87\%).

Os resultados sobre abundância, riqueza e diversidade de serpentes do Planalto Médio são pioneiros para o Rio Grande do Sul, revelando características importantes dessa comunidade de serpentes. Estudos de diversidade, dessa forma, podem contribuir com informações sobre a composição da fauna de serpentes e subsidiar futuros estudos de conservação para a área. Entretanto, há a necessidade da continuidade do monitoramento da fauna nas áreas estudadas, visando esclarecer a interferência das alterações do ambiente na estruturação da comunidade.

Revista Brasileira de Zoologia 23 (1): 211-217, março 2006 


\section{AGRADECIMENTOS}

À Corporação da Brigada Militar e ao Sr. Osvaldo Gomes, pela permissão no uso da fazenda para o desenvolvimento do trabalho. Á Gilvete Freitag, João V. Grando, Fernanda A. Gonçalves e acadêmicos do curso de Ciências Biológicas, pelo auxílio nas atividades de campo. À Márcia da S. Jorge, pela elaboração do mapa. Ao IBAMA (licença número 02/2002/RS) pela autorização concedida. À Otávio A.V. Marques e Ricardo J. Sawaya pelas sugestões.

\section{REFERÊNCIAS BIBLIOGRÁFICAS}

Accacio, G.M; A. Brant; R.M. Britez; R. Cerqueira; E.L.G. Espíndola; F. Godoy; E.C. Landau; A.T.L. Lopes; S.B. Mikich; N. Olifiers; B.V.S. Pimenta; O. Rocha; D.L. Silvano; W.S. Smith \& L.B. VENTORIn. 2003. Ferramentas biológicas para avaliação e monitoramento de habitats naturais fragmentados, p. 368389. In: Ministério do Meio Ambiente (Ed.). Fragmentação de ecossistemas. Causas, Efeitos sobre a biodiversidade e recomendações de políticas públicas. Brasília, Ministério do Meio Ambiente, 508p.

Barreto, I.L. \& I.L. Boldrini. 1990. Aspectos físicos, vegetação e problemática das regiões do Litoral, Depressão Central, Missões e Planalto do Rio Grande do Sul, p. 199-210. In: J.P. PUIGnAu (Ed.). Dialogo XXVIII. Montevidéu, II CA-Procesur, 379 p.

Cadle, J.E. \& H.W. Greene. 1993. Phylogenetic patterns, biogeography and the ecological structure of neotropical snake assemblages, p. 281-293. In: R.E. RickLeFes \& D. SCHLUter (Eds). Species diversity in ecological communities. Historical and geographical perspectives. Chicago, University of Chicago Press, 414p.

Cechin, S.Z. \& M. Martins. 2000. Eficiência de armadilhas de queda (pitfall traps) em amostragens de anfíbios e répteis no Brasil. Revista Brasileira de Zoologia, Curitiba, 17 (3): 729 740.

Cei, J.M. 1993. Reptiles del noroeste, nordeste y este de la Argentina. Herpetofauna de las servas subtropicales, Puna y Pampas. Torino, Museo Regionale di Scienze Naturali, 949p.

Colwell, R.K. 1997. EstimateS: statistical estimation of species richness and shared species from samples. Sunderland, Sinauer Associates, version 5.0.

Colwell, R.K. \& J.A. Coddington. 1994. Estimating terrestrial biodiversity through extrapolation. Philosophical transactions of the Royal Society, Series B, London, 345: 101118.

Dixon, J.R. 1989. A key and checklist to the neotropical snakes genus Liophis Wagler with country list and maps. Departament of Wildlife and Fisheries Sciences, Texas. Smithsonian Herpetological Informative, London, 79: 1-28.

Duellman, W.E. 1987. Lizards in an Amazonian rain forest community: Resource utilization and abundance. National
Geography Research, Washington, 3: 489-500.

EmBrapa. 1994. Macrozoneamento Agroecológico e Econômico. Porto Alegre, Empresa Brasileira de Pesquisa Agropecuária, 207p.

Giraudo, A. 2001. Serpientes de la Selva Paranaense y Del Chaco Húmedo. Buenos Aires, L.O.L.A. 328p.

Gotelli, N. J. \& R. Colwell. 2001. Quantifying biodiversity: procedures and pitfalls in the measurement and comparison of species richness. Ecology Letters, Montpellier, 4 (1): 379391.

Köppen, W. 1948. Climatologia. México, Fundo de Cultura Econômica, 478p.

LEMA, T. DE. 1994. Lista comentada dos répteis ocorrentes no Rio Grande do Sul. Comunicações Museu de Ciências e Tecnologia PUCRS, Porto Alegre, 7: 41-150.

Magurran, A.E. 1988. Ecological diversity and its measurement. Princeton, Princeton University Press, 179p.

Marques, O.A.V. \& I. SAZIma. 2004. História natural dos répteis da Estação Ecológica Juréia-Itatins, p. 212-236. In: O.A.V. Marques \& W. DulebA (Eds). Estação Ecológica Juréia-Itatins. Ambiente físico, flora e fauna. São Paulo, Holos, 384p.

Martins, M; M.S. Araújo; R.J. Sawaya \& R. Nunes. 2001. Diversity and evolution of macrohabitat use, body size and morphology in a monophyletic group of Neotropical pitvipers (Bothrops). Journal of Zoology, London, 254: 529-538.

Martins, M. \& E.M. Oliveira. 1998. Natural histoy of snakes in forests of the Manaus region, Central Amazonia, Brazil. Herpetological Natural History, Riverside, 6 (2): 78-150.

Peters, J.A \& B. Orejas-Miranda. 1970. Catalogue of the neotropical squamata: Part I. Snakes. Bulletin United States Natural Museun, Washington, 297: 1-347.

Prince, A.H. \& J.L. La Pointe. 1990. Activity patterns of a Chihuahuan desert snake community. Annals of Carnegie Museum, Pittsburgh, 59 (1): 15-23.

Ricklefs, R.E. 2003. A economia da natureza. Rio de Janeiro, Guanabara Koogan, 503p.

Quadros, F.L. \& V.P. Pillar. 1990. Transições floresta-campo no Rio Grande do Sul. Ciência e Ambiente, Santa Maria, 24: 109-118.

SAzimA, I. \& C.F.B. HADDAD. 1992. Répteis da Serra do Japi: notas sobre história natural, p. 212-231. In: L.P.C. Morellato (Eds). História natural da Serra do Japi. Ecologia e preservação de uma área florestal no sudeste do Brasil. Campinas, Editora da Unicamp, FAPESP, 321p.

SAzima, I. \& P.R. Manzani. 1995. As cobras que vivem numa reserva florestal urbana, p. 78-82. In: L.P.C. MoRellato \& H.F. LEITÃo-FILHo (Eds). Ecologia e preservação de uma floresta tropical urbana: Reserva de Santa Genebra. Campinas, Editora da Unicamp, 136p.

Scott, N.J. 1976. The abundante and diversity of the herpetofaunas of tropical litter. Biotropica, Washington, 8: 4-58.

Seigel, R.A. \& J.T. Collins. 1993. Snakes, ecology \& behavior. 
New York, Mc Graw-Hill Inc, 414p.

Seigel, R.A.; J.T. Collins \& S.S. NovaK. 1987. Snakes: ecology and evolutionary biology. New York, McGraw-Hill Publishing, 529p.

Strüssmann, C. 2000. Herpetofauna, p. 153-189. In: C.J.R. AlHo; P.N. CONCEIÇÃo; R. CONSTANTINO; T. SChLEMMERMEyer; C. STRÜSSMAnN; L.A.S. Vasconcellos; D.M.M. Oliveira \& M. Schneider (Eds). Fauna silvestre da região do rio Manso, MT. Brasília, Ibama, 267p.

Strüssmann, C. \& I. Sazima. 1993. The snake assemblage of the Pantanal at Poconé, western Brazil: Faunal composition and ecological summary. Studies on Neotropical Fauna and Environment, Lisse, 28 (3): 157-168.

Thompson, G.G.; P.C. Withers; E.R. Pianka \& S.A. Thompson. 2003. Assessing biodiversity whith species accumulation curves; inventories of small reptiles by pit-trapping in Western
Australia. Austral Ecology, Carlton, 28: 361-383.

Turner, F.B. 1977. The dynamics of populations of squamates and crocodilians, p. 157-264. In: C. GANS \& D.W. TINkLE (Eds). Biology of the Reptilia. New York, Academic Press, vol. 7, $682 \mathrm{p}$.

VitT, L.J. 1987. Communities, p. 335-365. In: R.A. Seigel; J.T. Colins; S.S. NOVAK (Eds). Snakes: ecology and evolutionary biology. New York, McGraw-Hill Publishing, 529p.

VitT, L.J. \& L.D. Vangilder.1983. Ecology of a Snake Community in Northeastem Brazil. Amphibia-Reptilia, Leiden, 4: 273296.

Yanosky, A.A.; J.R. Dixon \& C. Mercolli. 1996. Ecology of the snake community at el Bagual Ecological Reserve, Northeastern Argentina. Herpetological Natural History, Iowa, 4 (2): 97-110.

Anexo I. A lista de material examinado das espécies e o respectivo número de tombo.

\begin{tabular}{|c|c|}
\hline Espécies examinadas & Número de tombo \\
\hline Atractus thalesdelemai & CRUPF $837,897,948,1110,1126,1148$. \\
\hline Bothrops alternatus & $\begin{array}{l}\text { CRUPF } 706,713,716,772,789,841,889,894,913,914,918,919,929,930,962,964,974,975, \\
1000,1001,1003,1035,1049,1053,1064,1076,1104,1107,1108,1111,1115,1116,1137,1138, \\
1139,1140,1144,1165 .\end{array}$ \\
\hline Bothrops neuwiedi & CRUPF $812,928$. \\
\hline Chironius bicarinatus & CRUPF 717, 863, 917, 1030, 1067, 1078. \\
\hline Echinanthera cyanopleura & $\begin{array}{l}\text { CRUPF715, 771, 790, 792, 811, 822, 826, 857, 859, 860,862, 870, 872, 873, 874, 878, 882, 883, } \\
886,891,892,895,896,902,939,941,943,946,954,967,1004,1027,1031,1032,1036,1051, \\
1087,1102,1113,1129,1130,1173,1185 .\end{array}$ \\
\hline Echinanthera poecilopogon & CRUPF 976. \\
\hline Helicops infrataeniatus & CRUPF 971, 972, 978, 1070, 1184 \\
\hline Liophis flavifrenatus & CRUPF 757, 893, 910, 912, 966, 970, 973, 1045, 1075, 1131, 1132, 1167. \\
\hline Liophis jaegeri & CRUPF 869, 945, 1083, 1084, 1086, 1088, 1134, 1145. \\
\hline Liophis miliaris & CRUPF 890, 900, 960, 1117. \\
\hline Liophis poecilogyrus & $\begin{array}{l}\text { CRUPF } 804,823,827,836,840,887,921,923,924,925,940,944,949,950,952,953,963,987, \\
996,1028,1029,1033,1048,1073,1080,1096,1097,1100,1101,1103,1114,1161,1182 .\end{array}$ \\
\hline Micrurus altirostris & CRUPF 986. \\
\hline Oxyrhopus rhombifer & CRUPF 692, 693, 784, 988, 999, 1090, 1112, 1141, 1168, 1169, 1172, 1174, 1181, 1188, 1189. \\
\hline Philodryas aestiva & CRUPF 947, 1015, 1091, 1109, 1160. \\
\hline Philodyas patagoniensis & CRUPF 707, 791, 831, 865, 920, 968, 969, 977, 1050, 1052, 1143, 1149, 1163, 1166, 1175, 1186. \\
\hline Pseudablabes agassizii & CRUPF 691, 803, 824, 868, 916, 961, 985, 989, 1044, 1066. \\
\hline Thamnodynastes hypoconia & CRUPF 719. \\
\hline Thamnodynastes strigatus & $\begin{array}{l}\text { CRUPF } 688,689,694,714,719,721,810,825,828,832,832,834,839,856,861,864,867,871, \\
875,876,877,880,881,888,898,899,901,911,915,931,942,1002,1034,1065,1077,1085, \\
1089,1098,1125,1127,1128,1142,1147,1170,1171,1187,1268 .\end{array}$ \\
\hline Tomodon dorsatus & $\begin{array}{l}\text { CRUPF } 690,718,769,788,813,815,816,817,818,835,922,965,997,998,1046,1047,1069, \\
1074,1081,1099,1105,1106,1136,1146,1162,1164,11831190 .\end{array}$ \\
\hline
\end{tabular}

Recebido em 06.IV.2005; aceito em 22.II.2006. 